\title{
Seroprevalence of hepatitis B virus infection among antenatal clinic attendees at a tertiary hospital in Dar es Salaam, Tanzania
}

\author{
SABRIA RASHID ${ }^{1 *}$, CHARLES KILEWO² and SAID ABOUD ${ }^{3}$ \\ ${ }^{1}$ Department of Obstetrics and Gynaecology, Muhimbili National Hospital, P.O. Box 65000, Dar es Salaam, \\ Tanzania \\ ${ }^{2}$ Department of Obstetrics and Gynaecology, Muhimbili University of Health and Allied Sciences, Dar es Salaam, \\ Tanzania \\ ${ }^{3}$ Department of Microbiology and Immunology, Muhimbili University of Health and Allied Sciences, Dar es \\ Salaam, Tanzania
}

\begin{abstract}
Hepatitis B virus (HBV) infection is a serious public health problem in sub-Saharan Africa. The risk of vertical transmission increases if the mother is hepatitis B surface antigen (HBsAg) positive and more so when she is also hepatitis B envelope antigen ( $\mathrm{HBeAg}$ ) positive. Since 2000, the World Health Organization recommends screening of pregnant women for HBV infection. However, this is not currently practiced in Tanzania. The objective of this study was to determine seroprevalence and associated factors of HBV infection among pregnant women attending antenatal clinic at the Muhimbili National Hospital, in Dar es Salaam, Tanzania. This cross-sectional study was conducted from August-September 2010. Data on sociodemographic characteristics, obstetric and risk factors such as history of blood transfusion, and risky sexual practices was collected. Blood samples were tested for HBsAg, HBeAg, antibodies to HBsAg (Anti$\mathrm{HBs}$ ), IgM antibodies to hepatitis B core antigen (Anti-HBC) and anti-HIV antibodies. A total of 310 pregnant women (28.5 \pm 5.4 years) were enrolled in the study. Thirty-one percent (96) of the women were primigravidae. Most of studied women were of low educational status and married. The seroprevalence of HBsAg was 3.9\% (12/310) and none had detectable anti-HBs. None had IgM HBcAb, thus excluding acute $\mathrm{HBV}$ infection. All women tested negative for HBeAg. The overall seroprevalence of HIV infection was $9.7 \%$. Three of 12 (25\%) women had HBV-HIV co-infection. None of the assessed associated factors were significantly related to HBV infection. In conclusion, the seroprevalence of HBsAg among women attending antenatal care at Muhimbili National Hospital is moderate. It is recommended to introduce routine antenatal screening for HBV and "at birth dose" vaccination to new born babies of mothers found to be HBsAg positive.
\end{abstract}

Keywords: Hepatitis B virus, women, seroprevalence, antenatal clinic attendees, Tanzania

\section{Introduction}

The World Health Organization (WHO) considers hepatitis B virus (HBV) to be second to tobacco among the carcinogens causing malignancy (WHO, 2002). It is estimated that 2 billion people worldwide have been affected, of which 350 million people have chronic infection, and $10 \%$ of these are in sub-Saharan Africa and East Asia (WHO, 2002). These chronically infected ones may develop liver cirrhosis or hepatocellular carcinoma (WHO, 2002). Mother to child transmission is one of the major modes of spread of HBV. HBV-infected pregnant women are at risk of infecting their babies with a consequence of developing fulminant HBV infection especially when pregnant women are hepatitis $B$ surface antigen ( $\mathrm{HBsAg}$ ) positive, and more so when hepatitis $B$ e antigen (HBeAg) is also positive (Xu et al., 2002).

Tanzania was reported to be one of the countries with high endemicity levels (with seroprevalence rates higher than $8 \%$ ) of chronic HBV infection (WHO, 2002). In 1987, the HBsAg carrier rates in Dar es Salaam, Tanzania were estimated to be $10 \%$ and $15 \%$ among the general population and pregnant women, respectively (Haukenes et al., 1987). The seroprevalence of HBsAg among pregnant women in Tanzania was reported to vary between 3.5\% (Kibassa et al., 2004) and 6.3\% (Menendez et al., 1999). In a study conducted at the Muhimbili National Hospital the vertical transmission of HBV was found to be $11.8 \%$ (Kibassa et al., 2004). In Southern Tanzania

* Correspondence: Dr. Sabria Rashid; E-mail: sabriarashid@yahoo.com 
the reported perinatal transmission of HBV was 8\% (Menendez et al., 1999). Similarly, in a neighbouring country of Kenya data from Kenyatta National Hospital and eight Provincial hospitals suggested a persistent high prevalence of $\mathrm{HBsAg}$ carrier state among pregnant women with a figure of $9.3 \%$ in 2001/2002 (Okoth et al.,2006).

Vertical transmission of HBV can be prevented by immunization. Universal immunization has been recommended by the WHO since 1991 (WHO, 2002). In Tanzania, hepatitis B vaccination was introduced in 2002 in combination with diphtheria pertussis tetanus (DPT) at 4, 8 and 12 weeks of age of infants (MoHSW, 2007). A recent study by Metodi et al. (2010) reported that $69.3 \%$ of the immunized under-five children had protective anti-hepatitis B surface (anti-HBs) antibody levels. However, $1.7 \%$ of the children were found to be HBsAg positive suggesting occurrence of vertical transmission of HBV infection (Metodi et al., 2010).

In Nigeria, high parity, polygamy, multiple sexual partners and previous history of sexually transmitted diseases have been shown to be among the significant risk factors for HBV infection in pregnant women (Obi et al., 2006). In a study done at the Muhimbili National Hospital in Tanzania, the seroprevalence of HBV showed no association with marital status, previous history of jaundice, history of blood transfusion and age (Kibassa et al., 2004). Co-infection of HBV with human immunodeficiency virus (HIV) has been reported in $66.7 \%$ of the HIV-infected pregnant women in Dar es Salaam in 1989 (Shao et al. 1993). In other previous studies in Malawi, $71.7 \%$ were HBV-HIV co-infected while in Burkina Faso the HBV seroprevalence of $11.6 \%$ among HIV positive pregnant women were reported (Ahmed et al., 1998; Simpore et al., 2006). The reported increase in HBV prevalence over time and the influence of HIV substantiated the need for updated data in order to adopt the WHO recommendations of screening pregnant women and offering the atbirth-dose of HBV vaccine to prevent perinatal transmission. The objective of the current study was to determine the seroprevalence of HBV infection and associated factors among pregnant women attending antenatal clinic in a tertiary hospital in Dar es Salaam, Tanzania.

\section{Materials and Methods}

\section{Study design}

This was a cross-sectional study conducted at the antenatal care clinic (ANC) of Muhimbili National Hospital (MNH) in Dar es Salaam, Tanzania from August to September 2010. Pregnant women attending ANC were consecutively enrolled until the desired sample size was reached. $\mathrm{MNH}$ is a public tertiary referral hospital located in Dar es Salaam with a total bed capacity of 1400 patients. On average, 120 pregnant mothers attend the ANC on a daily basis.

\section{Data collection}

Information on the socio-demographic characteristics, previous history of blood transfusion and number of sexual partners were obtained from the study participants. All enrolled pregnant women had their HIV test results documented in their ANC cards, except three who were tested in the ANC during the study period. HIV testing was offered as a standard of care in the ANC. All pregnant mothers attending ANC except those who were immunized with hepatitis B vaccine were eligible for inclusion in the study.

\section{Laboratory investigations}

Blood specimens were collected for detection of $\mathrm{HBsAg}$, antibodies to hepatitis $B$ surface antigen (anti-HBs), IgM antibodies to hepatitis B core antigen (Anti-HBc), HBeAg and anti-HIV antibodies for pregnant women who had no HIV test results documented in their ANC cards. Laboratory testing for HBsAg was performed using microparticle enzyme immunoassay (MEIA) (Abbott AxSYM, Germany) in the Central Pathology Laboratory (CPL) at MNH. HBeAg testing was performed using an enzyme-linked fluorescent assay (VIDAS ${ }^{\circledR} \mathrm{HBe} / \mathrm{Anti}$-HBe system, Biomerieux, France). The anti-HBclgM antibodies as well as anti-HBs anti-HBsAb were determined using MEIA 
(Abbott AxSYM, Germany). HIV serostatus was determined using the National rapid HIV testing algorithm (Lyamuya et al., 2009). Samples were initially tested with SD HIV-1/2 3.0 Bioline followed by testing of reactive samples on Determine HIV-1/2 test. Sera that were reactive on both tests were considered HIV positive. Discordant results were resolved by a tiebreaker UniGold test.

\section{Data analysis}

Data were analyzed using the Statistical Package for Social Sciences (SPSS) version 16.0. Chisquare and Fishers' exact tests where applicable were performed to compare the proportions. The assessed dependent variable was the HBsAg serostatus. The independent variables were socio-demographic characteristics, history of blood transfusion and HIV serostatus. A p-value of $<0.05$ was regarded as significant.

\section{Ethical considerations}

The study received ethical approval from the Senate Research and Publications Committee of Muhimbili University of Health and Allied sciences. Permission to conduct the study was granted by $\mathrm{MNH}$ management. Written informed consent was obtained from the pregnant women prior to enrolment in the study. The pregnant women that were found to have HBV and/or HIV infection were referred to the respective clinics at $\mathrm{MNH}$ and managed according to the existing national HIV/AIDS treatment guidelines.

\section{Results}

A total of 310 pregnant women were enrolled in the study. They were from Kinondoni (131; $42.3 \%)$, Ilala (105; 33.9\%) and Temeke (70; 22.6\%) municipalities. Four were from other areas. The overall mean (SD) age was 28.5 (5.4) years. Of 310 pregnant women, $195(62.9 \%)$ were in the age group of 21-30 years. The majority of the women were married (86.8\%) and had primary level of education (51.0\%). Forty-five (14.5\%) women had other formal employment apart from health sector. Ninety-six (31.0\%) women were primigravidae. More than half of the women had one lifetime sexual partner (Table 1).

Table 1: Baseline socio-demographic characteristics of the study population $(\mathrm{N}=310)$

\begin{tabular}{llc}
\hline Characteristics & Response & $\mathbf{N}(\%)$ \\
\hline \multirow{3}{*}{ Age (years) } & $15-20$ & $17(5.5)$ \\
& $21-30$ & $195(62.9)$ \\
& Single & $98(31.6)$ \\
Marital status & Cohabiting & $22(7.1)$ \\
& Married & $18(5.8)$ \\
& Divorced & $269(86.8)$ \\
Level of education & Non-formal & $1(0.3)$ \\
& Primary & $3(1)$ \\
& Secondary & $158(51)$ \\
& College and University & $104(33.5)$ \\
Occupation & Health sector & $45(14.5)$ \\
& Petty trader & $23(7.4)$ \\
& Housewife & $89(28.7)$ \\
Parity & Other formal employment & $119(38.4)$ \\
& Others & $45(14.5)$ \\
& Non-employed & $14(4.5)$ \\
& o & $20(6.5)$ \\
\end{tabular}




$$
\begin{gathered}
1(0.3) \\
168(54.2) \\
119(38.4) \\
23(7.4)
\end{gathered}
$$

Twelve (3.9\%) of the pregnant women had positive HBsAg test results. None of the women had either detectable anti-HBs antibodies or IgM HBcAb. Furthermore, all these women tested negative for HBeAg. The overall seroprevalence of HIV infection was $9.7 \%$. Three of $12(25 \%)$ pregnant women had HBV-HIV co-infection. There were no significant differences in age, residence, marital status, education level, occupation and parity between those who tested

\begin{tabular}{|c|c|c|c|c|c|}
\hline \multirow[t]{2}{*}{ Characteristics } & Response & Total & $\begin{array}{l}\text { HBsAg +ve } \\
\mathrm{N}(\%)\end{array}$ & $\begin{array}{l}\text { HBsAg-ve } \\
\mathrm{N}(\%)\end{array}$ & P-value \\
\hline & $<20$ & 17 & - & $17(100)$ & \\
\hline \multirow[t]{3}{*}{ Age (years) } & $21-30$ & 195 & $10(5.1)$ & $185(94.9)$ & \\
\hline & $>30$ & 98 & $2(2)$ & $96(98)$ & 0.497 \\
\hline & Kinondoni & 131 & $5(3.8)$ & $126(96.2)$ & \\
\hline \multirow{4}{*}{ Residence } & Ilala & 105 & $3(2.9)$ & $102(97.1)$ & \\
\hline & Temeke & 70 & $4(5.7)$ & $66(94.3)$ & \\
\hline & Others & 4 & - & $4(100)$ & 0.701 \\
\hline & Single & 22 & - & $22(100)$ & \\
\hline \multirow{4}{*}{ Marital status } & Cohabiting & 18 & - & $18(100)$ & \\
\hline & Married & 275 & $12(4 \cdot 5)$ & $257(95.5)$ & \\
\hline & Divorced & 1 & - & $1(100)$ & 0.82 \\
\hline & Non-formal & 3 & - & $3(100)$ & \\
\hline \multirow{6}{*}{$\begin{array}{l}\text { Level } \\
\text { education }\end{array}$} & Primary & 158 & $5(3.2)$ & $153(96.8)$ & \\
\hline & Secondary & 104 & $6(5.8)$ & $98(94.2)$ & \\
\hline & University/College & 45 & $1(2.2)$ & $44(97.8)$ & 0.644 \\
\hline & Health sector & 23 & - & $23(100)$ & \\
\hline & Petty trader & 89 & $7(7.9)$ & $82(92.1)$ & \\
\hline & House wife & 119 & $1(0.8)$ & $118(99.8)$ & \\
\hline \multirow[t]{4}{*}{ Occupation } & Not employed & 20 & $1(5)$ & $19(95)$ & \\
\hline & $\begin{array}{l}\text { Formal } \\
\text { employment }\end{array}$ & 45 & $3(6.7)$ & $42(93.3)$ & \\
\hline & Others & 14 & - & $14(100)$ & 0.073 \\
\hline & 0 & 96 & $4(4.2)$ & $92(95.8)$ & \\
\hline \multirow[t]{2}{*}{ Parity } & $1-5$ & 213 & $8(3.8)$ & $205(96.2)$ & \\
\hline & $\geq 6$ & 1 & - & $1(100)$ & 1.00 \\
\hline
\end{tabular}
positive and those who tested negative to HBsAg (Table 2).

Table 2: Socio-demographic characteristics in relation to HBsAg serostatus of the study population $(\mathrm{N}=310)$.

No statistically significant differences were observed in the number of life-time sexual partners, HIV serostatus and receipt of blood transfusion between those with positive and those with negative HBsAg serostatus (Table 3). 
Table 3: Sexual history, HIV sero-status and history of blood transfusion in relation to $\mathrm{HBsAg}$ serostatus of the study population $(\mathrm{N}=310)$

\begin{tabular}{llllll}
\hline Characteristics & Response & $\begin{array}{l}\text { Total } \\
(\mathbf{1 0 0 \%})\end{array}$ & $\begin{array}{l}\text { HBsAg +ve } \\
\mathbf{N}(\%)\end{array}$ & $\begin{array}{l}\text { HBsAg -ve } \\
\mathbf{N}(\%)\end{array}$ & P-value \\
No. of Sexual partners & 1 & 168 & $4(2.4)$ & $164(97.6)$ & \\
& $2-3$ & 119 & $7(5.9)$ & $112(94.1)$ & \\
HIV sero-status & 24 & 23 & $1(4.3)$ & $22(95.7)$ & 0.239 \\
\multirow{4}{*}{ History of blood } & Positive & 30 & $3(10)$ & $27(90)$ & \\
transfusion & Negative & 280 & $9(3.2)$ & $271(96.8)$ & 0.099 \\
& Yes & $36^{*}$ & - & $36(100)$ & \\
& No & 274 & $12(4.4)$ & $262(95.6)$ & 0.373
\end{tabular}

*NB: Of these 36 women, 25 (69.4\%) received blood transfusion once; while the remaining had two or more blood transfusions.

\section{Discussion}

The seroprevalence of HBsAg in this study is in agreement with findings from a previous study among pregnant women presenting at the labour ward in the same hospital (Kibassa et al., 2004). In the previous study, it was found that the overall prevalence of HBsAg in Dar es Salaam city was similar to that found at Muhimbili National Hospital (Kibassa et al., 2004). According to $\mathrm{WHO}$, the $3.9 \%$ prevalence found in this study is regarded as of moderate level of HBV infection. WHO defines low prevalence to be $<2 \%$, moderate prevalence as $2-8 \%$, and high prevalence as $>8 \%$ $\mathrm{HBsAg}$ positivity. The WHO therefore recommends universal immunization of all infants to be adopted by all countries irrespective of HBsAg prevalence (WHO, 2002).

The findings of the current study indicate that the seroprevalence of HBsAg at MNH has remained fairly stable over the past 14 years. This relatively stable seroprevalence of HBsAg found among pregnant women over time could possibly be explained by, firstly, the routine HIV and syphilis screening during antenatal period and strengthened campaigns against HIV, a disease which has a shared mode of transmission with HBV. A decrease in new HIV infections in Tanzania among people aged 15 years and above from 91,000 in 2001 to 69,000 in 2012 has been reported (UNAIDS, 2013). The other possible reason for the observed stable seroprevalence of $\mathrm{HBsAg}$ could be the fact that all donated blood throughout the country is presently subjected to rigorous screening measures for HIV and HBV infections and thus reducing the risk of HBV transmission to recipients (MoHSW, 2006). The stable seroprevalence of HBsAg could also be due to the use of antiretroviral therapy (ART) regimens that contain lamivudine (Su et al., 2004; Gambarin-Gelwan et al., 2007). Use of ART among HIV-infected patients of whom the majority were women at the MNH has shown that there is a high adherence to ART (Mugusi et al., 2009) and improved access to HIV care and treatment (Mugusi et al., 2010),

Despite the finding of a relatively stable seroprevalence of HBsAg in Dar es Salaam, higher prevalence has been reported from a neighbouring country of Kenya where a prevalence of 9.3\% was found among pregnant women (Okoth et al., 2006). Factors that were described to lead to a relatively higher seroprevalence in Kenya included low socio-economic status and female genital mutilation. Previous studies conducted at $\mathrm{MNH}$ reported higher HBV seroprevalence levels among other populations. The prevalence among blood donors was reported to be $11 \%$ (Matee et al., 2008). On the other hand, the HBV prevalence among HIVinfected patients in 2006 was reported to be 17.3\% (Nagu et al., 2008). The national HBV prevalence among blood donors ranged from 6.1\% to 4.9\% between 2009 and 2010 (MoHSW, 2011). The differences from the present study are probably due to the populations studied which are inherently different. HBV infection is predominantly a problem in the resource-limited countries. Infection with HBV among pregnant women has been reported in other African countries including Nigeria (6.8\%) (Rabiu et al., 2010) and Libya (1.5\%) (El-Magrahe et al., 2010). 
It was found out in the current study that all of the 12 women who were HBsAg positive were $\mathrm{HBeAg}$ negative. It is known that presence of $\mathrm{HBeAg}$ is an important indicator of transmissibility of HBV infection from the mother to the baby (WHO, 2002). Therefore, all these women had a low probability of infecting their newborn babies or their sexual partners. Additionally, the presence of antibodies against $\mathrm{HBeAg}$ is known to indicate that the person is not infectious at all (WHO, 2002). However, this was not confirmed in our study due to limited resources.

Reported rates of vertical transmission of HBV and seroprevalence of HBeAg in Tanzania and neighbouring countries are varied. The study by Kibassa et al. (2004) in Dar-es-salaam reported the rate of vertical transmission of HBV to be $11.8 \%$, but the presence of HbeAg was not detected. A study in Kenya reported the prevalence of HBeAg of 8.8\% (Okoth et al., 2006). On the other hand, another previous study on HBV markers in pregnant women in Dar-es-Salaam in 1975 reported that none of the HBsAg positive pregnant women was positive for $\mathrm{HBeAg}$ (done by Haukenes et al. (1978) similar to the findings of the current study. Chronic carrier state of HBV infection has been associated with the finding of negative HBeAg (WHO, 2002). On the other hand, the detection of $\mathrm{HBeAg}$ increases the chance of transmissibility of HBV. However, the lack of detection of HBeAg per se should not be taken as indicating total lack of HBV transmissibility. Rather, the presence of HBV infection in pregnant women can lead to the spread and acquisition of infection to newborn babies. It is reported that perinatally acquired infections account for about $90 \%$ of all cases of HBV globally, and can lead to a chronic carrier state and other long-term sequelae (WHO, 2002). It was also found in this study that none of the women had anti-HBsAg antibodies indicating that these were chronically infected.

In order to prevent chronic HBV and hepatocellular carcinoma, WHO has recommended universal immunization. In Tanzania, vaccination against HBV infection at infancy stage was introduced in 2002 and has been continuing since then (MoHSW, 2007). Despite these efforts, a study done by Metodi et al. (2010) reported that only about two thirds of the underfives had protective antibodies post immunization, and $1.7 \%$ of them were found to be HBsAg positive signifying that perinatal transmission occurs despite the immunization given. These findings from the study by Metodi et al (2010), and the findings of the current study that HBsAg seroprevalence is still of moderate intensity suggest that it will be beneficial to have routine screening of mothers during the antenatal period so that appropriate actions are taken timely.

In this study none of the HBsAg positive pregnant women had IgM form of HBCAb, implying that none had acute HBV infection. The $\lg M$ form of $\mathrm{HBCAb}$ is present during acute infection and tends to disappear approximately 6 months after infection (WHO, 2002). It is therefore difficult to determine the time point at which these pregnant women became infected with the HBV. A quarter of the pregnant women with HBsAg were found co-infected with HIV. Similar HBV-HIV co-infection has been reported previously among pregnant women in Tanzania (Shao et al., 1993) and elsewhere (Ahmed et al., 1998; Santiago-Munoz et al., 2005; Simpore et al., 2006; Nagu et al., 2008).

Despite an attempt by this study, no clear risk factors including HIV associated with HBV infection could be established. This is because the study was not powered to demonstrate this. The study was limited by the fact that it is a hospital-based study and that pregnant women attending ANC at this tertiary hospital were a select population of those likely to have obstetric complications hence, the results can't be generalized for the whole country. Furthermore, the exclusion of private patients who pay for services offered could also affect the generalization of findings. This was however minimized by the fact that non-paying patients were a mixture of pregnant women from low as well as high socio-economic status. However, the current study findings have shed light into the problem of HBV infection among pregnant mothers in the local settings. In conclusion, the seroprevalence of $\mathrm{HBsAg}$ observed in the current study is of moderate severity according to WHO endemicity levels of HBV. It is recommended to introduce 
routine antenatal screening for HBV and "at birth dose" vaccination to new born babies of mothers found to be HBsAg positive.

\section{Conflict of interest}

The authors have no competing interests to declare.

\section{Acknowledgements}

We would like to thank all the nursing and laboratory technologists at the Ante-natal care clinic and in the Microbiology Section of the Central Pathology Laboratory, Muhimbili National Hospital, for data collection and laboratory testing, respectively.

\section{References}

Ahmed, S.D., Cuevas, L.E., Brabin, B.J., Kazembe, P., Broadhead, R., Verhoeff, F.H. \& Hart, C.A. (1998) Seroprevalence of hepatitis B and C and HIV in Malawian pregnant women. Journal of Infection 37, 248-251.

El-Magrahe, H., Furarah, A.R., El-Figih, K., El-Urshfany, S. \& Ghenghesh, K.S.,(2010) Maternal and neonatal seroprevalence of Hepatitis B surface antigen (HBsAg) in Tripoli, Libya. Journal of Infection in Developing Countries 4, 168-170.

Gambarin-Gelwan, M. (2007) Hepatitis B in pregnancy. Clinics in Liver Disease 11, 945-963.

Haukenes, G., Shao, J., Mbena, E. \& Rustad, S. (1987) Hepatitis B virus markers in the population of Dar-es-salaam, Tanzania. Journal of Infection 15, 183-188.

Kibassa, C.J. \& Msengi, A.E. (2004) Determination of vertical transmission of hepatitis B virus in Dar es Salaam. Tanzania Medical Journal 19, 21-25.

Lyamuya, E.F., Aboud, S., Urassa, W.K., Sufi, J., Mbwana, J., Ndugulile, F. \& Massambu, C. (2009) Evaluation of simple rapid HIV assays and development of national rapid HIV test algorithms in Dar es Salaam, Tanzania. BMC Infectious Diseases 18, 9-19.

Matee, M.I., Magesa, P.M. \& Lyamuya, E.F. (2006) Seroprevalence of human immunodeficiency, hepatitis $B$ and $C$ viruses and syphilis infections among blood donors at Muhimbili National Hospital in Dar es Salaam, Tanzania. BMC Public Health 30, 6-21.

Menendez, C., Sanchez-Tapias, J.M., Kahigwa, E., Mshinda, H., Costa, J., Vidal, J., Acosta, C., Lopez-Labrador, X., Olmedo, E., Navia, M., Tanner, M., Rodes, J. \& Alonso, P.L. (1999) Prevalence and mother-to-infant transmission of hepatitis viruses B, C, and E in Southern Tanzania. J Medical Virolology 58, 215-220.

Metodi, J., Aboud, S., Mpembeni, R. \& Munubhi, E. (2010) Immunity to hepatitis B vaccine in children under five years attending reproductive and child health clinic in Dar es Salaam, Tanzania. Annals of Tropical Paediatrics 30, 129-136.

MoHSW (2006) Guidelines on the Clinical Use of Blood and Blood Products. Ministry of Health and Social Welfare, Dar es Salaam, Tanzania.

MoHSW (2007) Expanded Programme on immunization 2006-2010, Tanzania. Comprehensive Multiyear Plan, 2nd edition. Ministry of Health and Social Welfare, Dar es Salaam, Tanzania, Government Press, 5-7.

MoHSW (2011) HIV/AIDS/STI Surveillance Report No. 22. National AIDS Control Programme, Ministry of Health and Social Welfare, Dar es Salaam, Tanzania.

Mugusi, F., Mugusi, S., Bakari, M., Hekdemann, B., Josiah, R., Janabi, M., Aboud, S., Aris, E., Swai, H., Mhalu, F., Biberfeld, G., Pallangyo, K. \& Sandstrom E. (2009) Enhancing adherence to Antiretroviral therapy at the HIV clinic in Resource constrained countries; the Tanzanian Experience. Tropical Medicine \& International Health 14, 1226-1232. 
Mugusi, S.F., Mwita, J.C., Francis, J.M., Aboud, S., Bakari, M., Aris, E.A., Swai, A.B., Mugusi, F.M., Pallangyo, K., \& Sandstrom, E. (2010) Effect of Improved access to antiretroviral therapy on clinical characteristics of patients enrolled in the HIV care and treatment clinics at Muhimbili National Hospital (MNH), Dar es Salaam, Tanzania. BMC Public Health 10, 291.

Nagu, T.J., Bakari, M. \& Matee, M.I. (2008) Hepatitis A, B and C viral co-infections among HIV infected adults presenting for care and treatment at Muhimbili National Hospital in Dar es Salaam, Tanzania. BMC Public Health 8, 416.

Obi, S.N., Onah, H.E., \& Ezugwu, F.O. (2006) Risk factors for hepatitis B infection during pregnancy in a Nigerian obstetric population. Journal of Obstetrics and Gynaecology 26, 770-772.

Okoth, F., Mbuthia, J., Gatheru, Z., Murila, F., Kanyingi, F., Mugo, F., Esamai, F., Alavi, Z., Otieno, J., Kiambati, H. \& Wanjuki N. (2006) Seroprevalence of hepatitis B markers in pregnant women in Kenya. East African Medical Journal 83, 485-493.

Rabiu, K.A., Akinola, O.I., Adewunmi, A.A., Omololu, O.M. \& Ojo, T.O. (2010) Risk factors for hepatitis $B$ virus infection among pregnant women in Lagos, Nigeria. Acta Obstetricia et Gynecologica Scandinavica 89, 1024-1028.

Santiago-Munoz, P., Roberts, S., Sheffield, J., McElwee, B. \& Wendel, G.D. Jr. (2005) Prevalence of hepatitis $B$ and $C$ in pregnant women who are infected with human immunodeficiency virus. American Journal of Obstetrics and Gynecology 193, 1270-1273.

Shao, J.F., Haukenes, G., Yangi, E. \&Vollset, S.E. (1993) Association of hepatitis B and human immunodeficiency virus infections in Tanzanian population groups. European Journal of Clinical Microbiology and Infectious Diseases 12, 62-64.

Simpore, J., Savadogo, A., Ilboudo, D., Nadambega, M.C., Esposito, M., Yara, J., Pignatelli, S., Pietra, V. \& Musumeci, S. (2006) Toxoplasma gondii, HCV and HBV seroprevalence and coinfection among HIV-positive and -negative pregnant women in Burkina Faso. Journal of Medical Virology 78, 730-733.

Su, G.G., Pan, K.H., Zhao, N.F., Fang, S.H., Yang, D.H. \& Zhou, Y. (2004) Efficacy and safety of lamivudine treatment for chronic hepatitis $\mathrm{B}$ in pregnancy. World Journal of Gastroenterology 15, 910-912.

UNAIDS (2013) UNAIDS Report on the global AIDS Epidemic http://www.unaids.org/en/media/unaids/contentassets/documents/epidemiology/2013/gr 2013/UNAIDS_Global_Report. Accessed $19^{\text {th }}$ October 2011

WHO (2002) Hepatitis B. Horld Organization.www.who.int/entity/csr/disease/hepatitis/HepatitisB_whocdscsrly02002_2.p df.Accessed $19^{\text {th }}$ October 2011

Xu, D.Z., Yan, Y.P., Choi, B.C., Xu, J.Q., Men, K., Zhang, J.X., Liu, Z,H. \& Wang, F.S. (2002) Risk factors and mechanism of transplacental transmission of hepatitis $B$ virus: a case-control study. Journal of Medical Virology 67, 20-26. 